\title{
Desenvolvimento de um Chatbot baseado em Ontologia para Atendimento a Chamados de Suporte ao Cliente
}

\author{
Malcom F. B. Silva ${ }^{1}$, Cristiane A. Yaguinuma ${ }^{1}$, Fábio J. J. dos Santos ${ }^{1}$, Tales Boalim ${ }^{2}$ \\ ${ }^{1}$ Instituto Federal de São Paulo (IFSP) - Câmpus Araraquara \\ Rua Doutor Aldo Benedito Pierri, 250 - Araraquara - SP \\ ${ }^{2} \mathrm{JN}$ Moura Informática \\ Av. Cristovão Colombo, 2264 - Araraquara - SP \\ malcom.s@aluno.ifsp.edu.br, cristiane.yaguinuma@ifsp.edu.br \\ fabio.jjseifsp.edu.br, tales@rfidmoura.com.br
}

\begin{abstract}
Resumo. Chatbots no contexto de suporte ao cliente permitem que dúvidas frequentes sejam resolvidas de forma rápida sem necessitar a alocação de um atendente e, consequentemente, podendo aumentar a quantidade de atendimentos por dia. Foi feito um estudo de caso de uma empresa sobre a senha de liberação do sistema, um pedido que causava sobrecarga na equipe de suporte. $O$ objetivo do projeto foi desenvolver um chatbot capaz de, em um primeiro momento, responder os pedidos sobre senha de liberação e reduzir a quantidade de atendimentos do suporte humano. Foram realizados estudos de diversas ferramentas para desenvolvimento do chatbot e a ferramenta escolhida para o projeto foi a Dialogflow por possuir todas as funcionalidades necessárias para a implementação. Foram realizados experimentos com usuários, apresentando resultados satisfatórios.
\end{abstract}

\section{Introdução}

Chatbots são programas de computador que interagem com usuários utilizando língua natural [Shawar and Atwell 2007]. Tais sistemas têm sido utilizados nos mais diferentes domínios, atuando diversas vezes como sistemas especialistas. Geralmente são encontrados chatbots em serviços de atendimento ao consumidor devido ao fato da maioria dos pedidos dos clientes ser concentrada em um número reduzido de diferentes tipos de requisições. A grande demanda gerada pelos clientes pode causar sobrecarga do serviço de suporte e consequentemente levar a longas esperas até o atendimento ser realizado. Ao utilizar um chatbot, os atendimentos mais simples podem ser resolvidos mais rapidamente além de reduzir a demanda no suporte convencional.

Dentre as plataformas de chatbot disponíveis, foram pesquisadas [DIALOGFLOW 2018], [WATSON 2018] e [WITAI 2018]. Nas plataformas estudadas há quatro conceitos fundamentais: Intents, Entities, Contexts e Web hooks. Intents representam o assunto da mensagem e são definidas como uma lista de frases que o usuário poderia digitar para determinado assunto ("Qual é a previsão do tempo?" pode ser identificado como intent "clima", por exemplo). Entities são utilizadas para interpretar variações nos intents (em "Vai chover hoje?" e "Vai chover amanhã?" as palavras "hoje" e "amanhã" podem fazer parte da entity "dia" com ambas as frases no intent "chuva"). Contexts permitem relacionar o intent atual com os anteriores (se o usuário escrever "e 
semana que vem?" é possível relacionar com o intent anterior "chuva" e saber que o usuário está perguntando se vai chover semana que vem). Web hooks são uma forma de fazer chamadas a um serviço em um servidor externo para executar lógica de negócio ao identificar certo intent (ao perguntar se vai chover, o chatbot pode chamar um serviço de previsão do tempo para obter os dados necessários e retornar uma resposta útil ao usuário). Wit.ai possui intents e entities, mas não possui contexts e web hooks. Watson oferece intents e contexts além de possuir web hooks, porém são restritos ao Bluemix, serviço de nuvem da IBM. Dialogflow dispõe de intents, contexts e web hooks não restritos a um serviço específico. Sendo assim, a plataforma escolhida para o projeto foi a Dialogflow.

Além de identificar o assunto de determinada mensagem, também há a necessidade de usar vocabulário estruturado para tratar alguns tipos de atendimento do chatbot. Por exemplo, tal vocabulário poderia ser utilizado ao pedir ajuda para configurar uma balança de determinada marca. Cada marca possui métodos distintos para configuração e consequentemente a resposta do chatbot será distinta para cada marca. Para representar este vocabulário, pode ser utilizada uma ontologia a fim de identificar termos relacionados e oferecer um melhor entendimento da frase. Segundo [Al-Zubaide and Issa 2011], ontologias são legíveis por humanos, compreensíveis, compartilháveis e formais, ou seja, são expressadas em uma linguagem que possui semântica bem definida. Por meio de ontologias é possível realizar uma modelagem genérica e extensível para utilização no chatbot.

Neste sentido, este artigo descreve o desenvolvimento de uma aplicação de chatbot utilizando ontologia e a plataforma [DIALOGFLOW 2018] com o intuito de reduzir a quantidade de atendimentos realizados pela equipe de suporte e integrá-lo ao serviço de suporte de uma empresa. Após a identificação das principais necessidades e a definição dos requisitos do projeto, foi definido que o chatbot iria atuar inicialmente nos atendimentos relacionados a senha de acesso dos sistemas e posteriormente tratar outros tipos de atendimentos que considerem variações de vocabulário com base em ontologias. Para avaliar a eficácia da solução foram coletadas estatísticas de uso do chatbot pela empresa além de experimentos com funcionários da empresa.

O restante deste artigo está organizado da seguinte forma. A Seção 2 explica os protótipos desenvolvidos e a arquitetura do chatbot. O experimento realizado com usuários reais é apresentado na Seção 3. Por fim, a Seção 4 contém as considerações finais e intenções de trabalhos futuros.

\section{Metodologia e Desenvolvimento}

Esta seção contém detalhes do desenvolvimento do chatbot, que resultou na construção de dois protótipos. O primeiro protótipo não utiliza ontologia e foi desenvolvido para fins de coleta de requisitos e aprendizado com a plataforma Dialogflow. O segundo protótipo possui integração com ontologia para sugestões de resposta. Na empresa está sendo utilizada uma versão modificada do primeiro protótipo.

\subsection{Protótipo Inicial}

O estudo de caso considerou temas específicos de atendimento do suporte da referida empresa. Foram realizadas reuniões para que fossem coletados os requisitos e o escopo 
do projeto. Assim, definiu-se que o chatbot iria atuar inicialmente no escopo de senha de liberação. Tal atendimento pode ser efetuado pelo chatbot devido à baixa complexidade da pergunta e do sistema de geração de senha ser automatizado. Há duas categorias na qual um usuário pode ser classificado ao requisitar a senha de liberação. O usuário está na primeira categoria quando a situação financeira está em dia e pode receber a senha. Usuários que possuem pendências financeiras e, consequentemente, não podem receber a senha se enquadram na segunda categoria.

Com o escopo e plataforma definidos, foi desenvolvido um protótipo inicial utilizando apenas funcionalidades básicas, ou seja, não foi adicionado suporte a ontologia pois o objetivo no momento era estudar a plataforma Dialogflow e desenvolver uma solução inicial para o escopo de senha de liberação.

A arquitetura consiste em um intent para senha de liberação contendo trechos de atendimentos anteriores relacionados ao assunto a fim de treinar o chatbot a entender frases similares. A Fig. 1 mostra a interface do Dialogflow com algumas frases de treinamento adicionadas manualmente a partir de conversas arquivadas do suporte online da empresa.

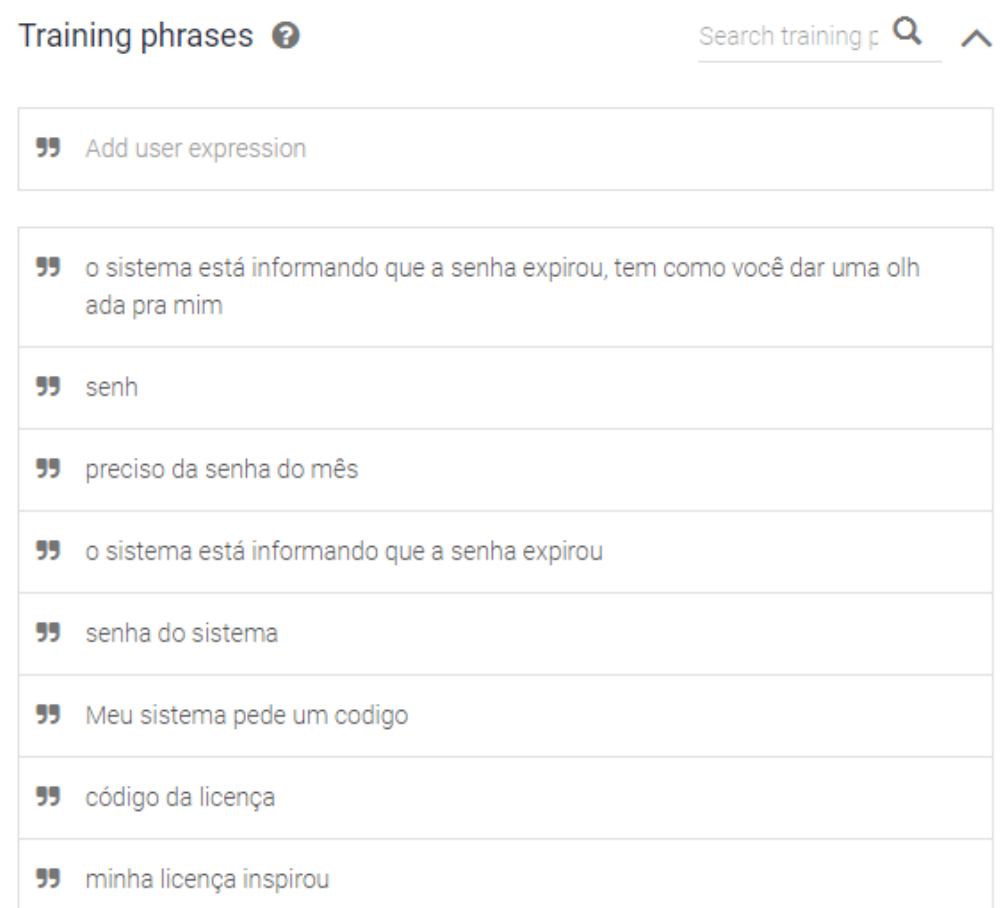

Figura 1. Interface para frases de treinamento.

O protótipo desenvolvido é capaz de compreender frases relacionadas ao pedido de senha de liberação e enviar uma requisição a um webservice da empresa para obter a situação financeira do usuário. Provido de tal informação, o protótipo responde ao usuário de acordo com uma das duas situações possíveis citadas anteriormente, ou seja, liberando a senha de acesso do mês ou orientando o usuário a entrar em contato com o setor financeiro para esclarecimentos. A Fig. 2 mostra o protótipo desenvolvido com um exemplo de conversa sobre senha de liberação. Antes de acessar o chat o usuário deve informar o CNPJ da empresa ou o próprio CPF para fins de identificação do sistema 
contratado pela empresa. A identificação do usuário é necessária para que possa verificar o status financeiro e gerar a senha de liberação.

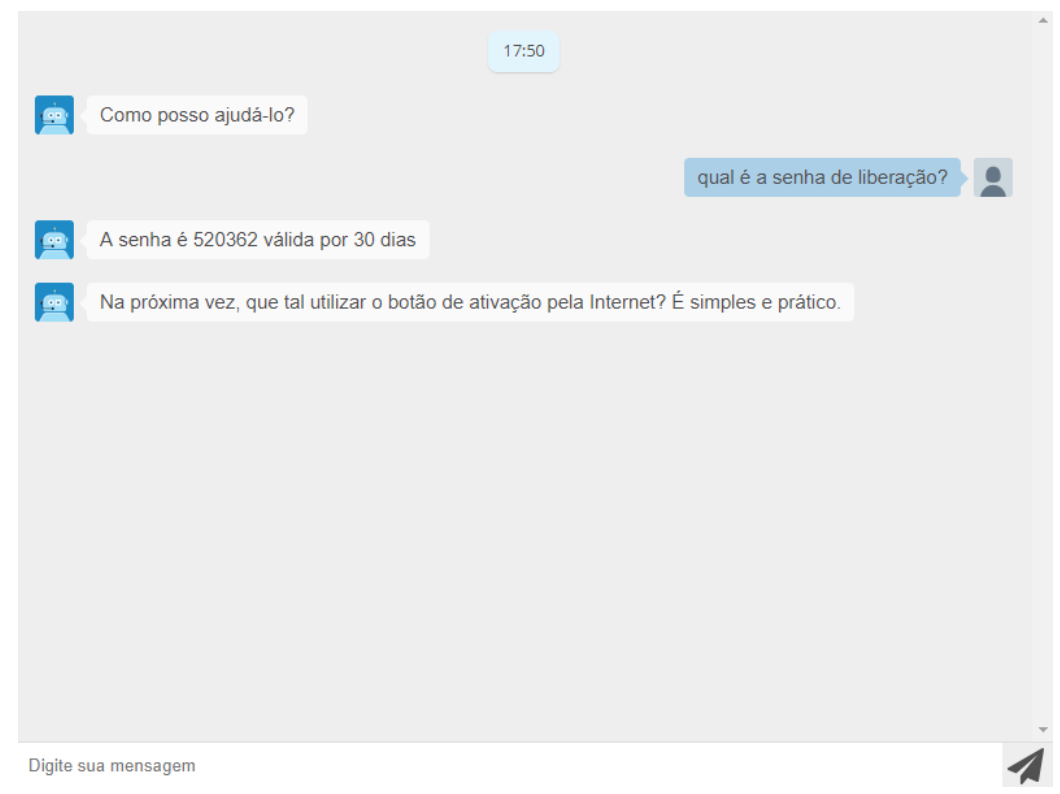

Figura 2. Interface gráfica do protótipo inicial desenvolvido.

O protótipo foi apresentado à empresa para avaliação. Após os ajustes necessários, o chatbot foi integrado ao sistema de suporte online. A Fig. 3 mostra a sequência de ações que ocorrem quando um usuário envia uma mensagem.

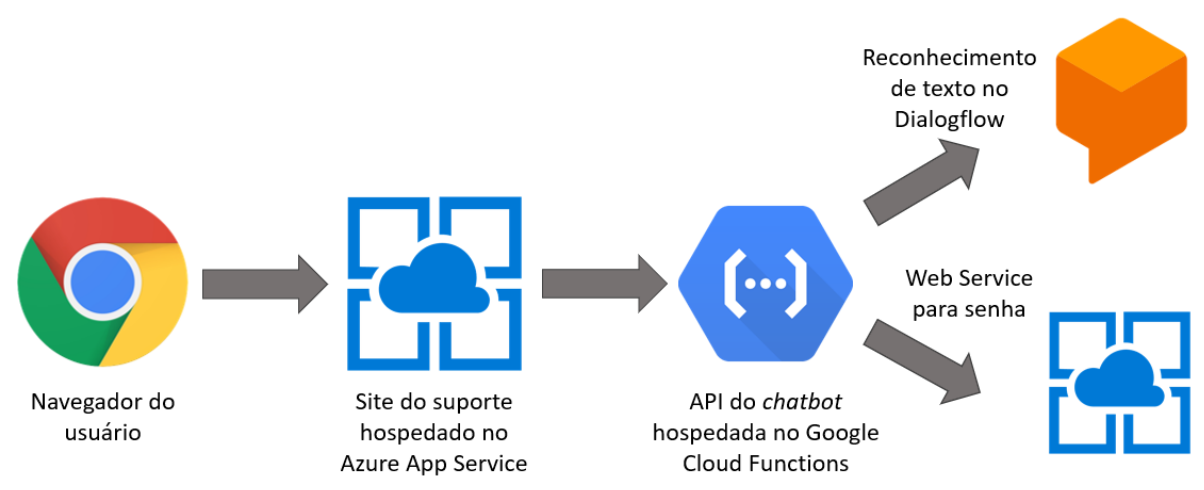

Figura 3. Diagrama em alto-nível da implementação.

Durante o primeiro mês de operação no suporte da empresa foi contabilizada a quantidade de mensagens relacionada à senha de liberação. Das mensagens contadas $81,52 \%$ foram identificadas corretamente, $16,31 \%$ não foram identificadas (falso negativo) e $2,17 \%$ foram identificadas incorretamente (falso positivo). Um gráfico das porcentagens pode ser visto na Fig. 4. Os resultados obtidos com o protótipo inicial foram publicados por [Silva et al. 2018] .

\subsection{Chatbot baseado em ontologia}

O suporte a ontologia foi desenvolvido utilizando o framework Jena [Carroll et al. 2004]. A interface com o servidor do chatbot utiliza webservices. A Fig. 5 contém uma 


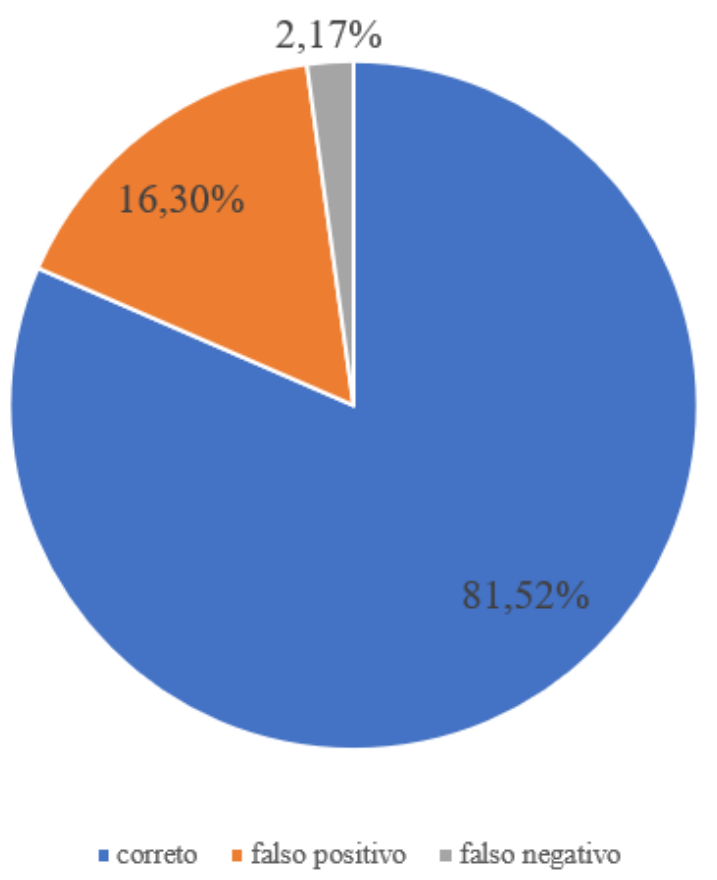

Figura 4. Gráfico de mensagens identificadas.

representação simplificada da sequência de interações realizadas. O usuário envia a mensagem na página de suporte, o servidor encaminha o pedido para a plataforma Dialogflow, a resposta é processada e caso seja sobre senha de liberação, o servidor obtém a senha por meio do webservice da empresa. Por fim o servidor envia um pedido para o servidor de ontologia para obter as sugestões de resposta para aquela mensagem.

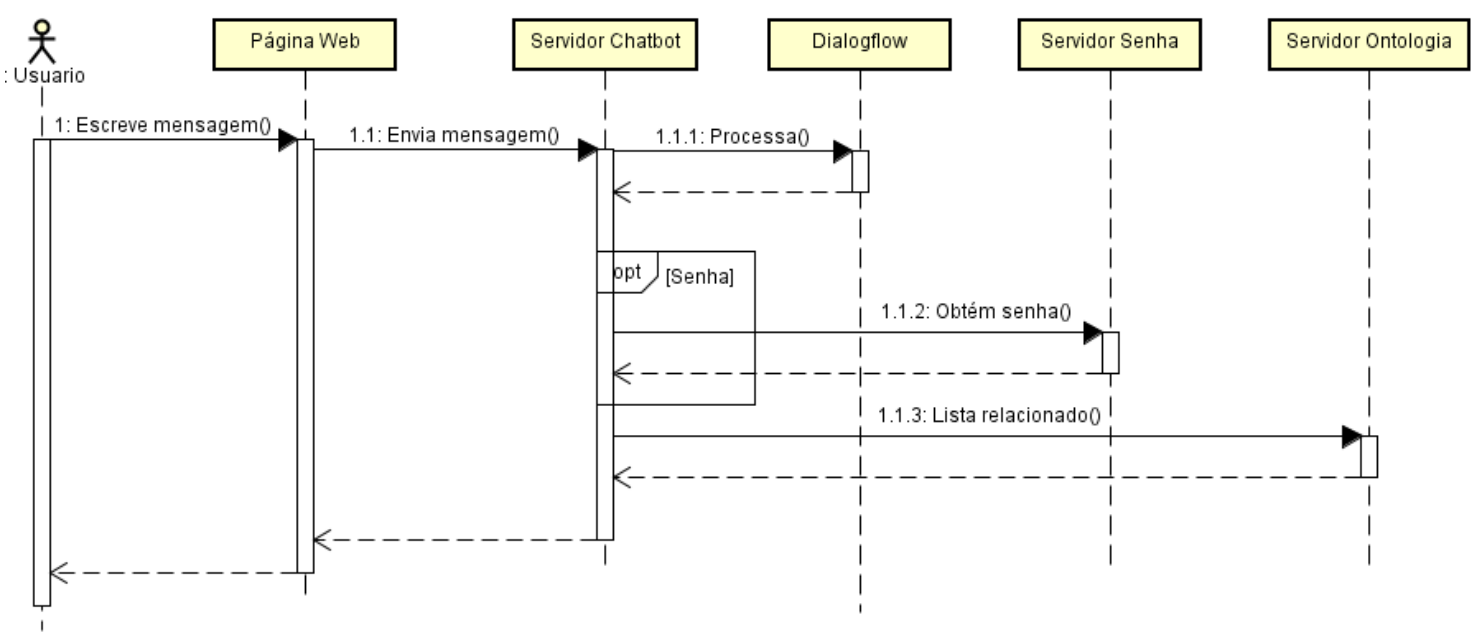

Figura 5. Diagrama de sequência do chatbot baseado em ontologia.

Como pode ser visto na Fig. 6, a interface gráfica foi alterada para mostrar botões acima do campo de escrita de mensagem. Os botões contêm o resultado da pesquisa no webservice de ontologia. Ao clicar no botão uma nova mensagem é enviada com o texto do botão. 
$\because \quad$ Como posso ajudá-lo?

Figura 6. Interface gráfica do protótipo desenvolvido com sugestão de resposta.

A modelagem da ontologia foi feita utilizando a ferramenta Protégé [Knublauch et al. 2004] utilizando dados do primeiro protótipo utilizado na empresa. Os temas mais recorrentes no suporte online da empresa foram utilizados como casos iniciais para a modelagem manual das classes. Na Fig. 7 pode-se observar as classes modeladas e os atributos da classe Boleto. As classes são agrupadas por temas e cada classe está vinculada a um intent do Dialogflow por meio do atributo intent, ou seja, se o usuário digitar algo relacionado a boleto e o Dialogflow retornar o intent bot.boleto, a interface exibirá todos os filhos diretos da classe cujo atributo intent for bot.boleto, que no caso da Fig. 7 seria Sim e Não. O texto que será exibido para o usuário é armazenado no atributo label.

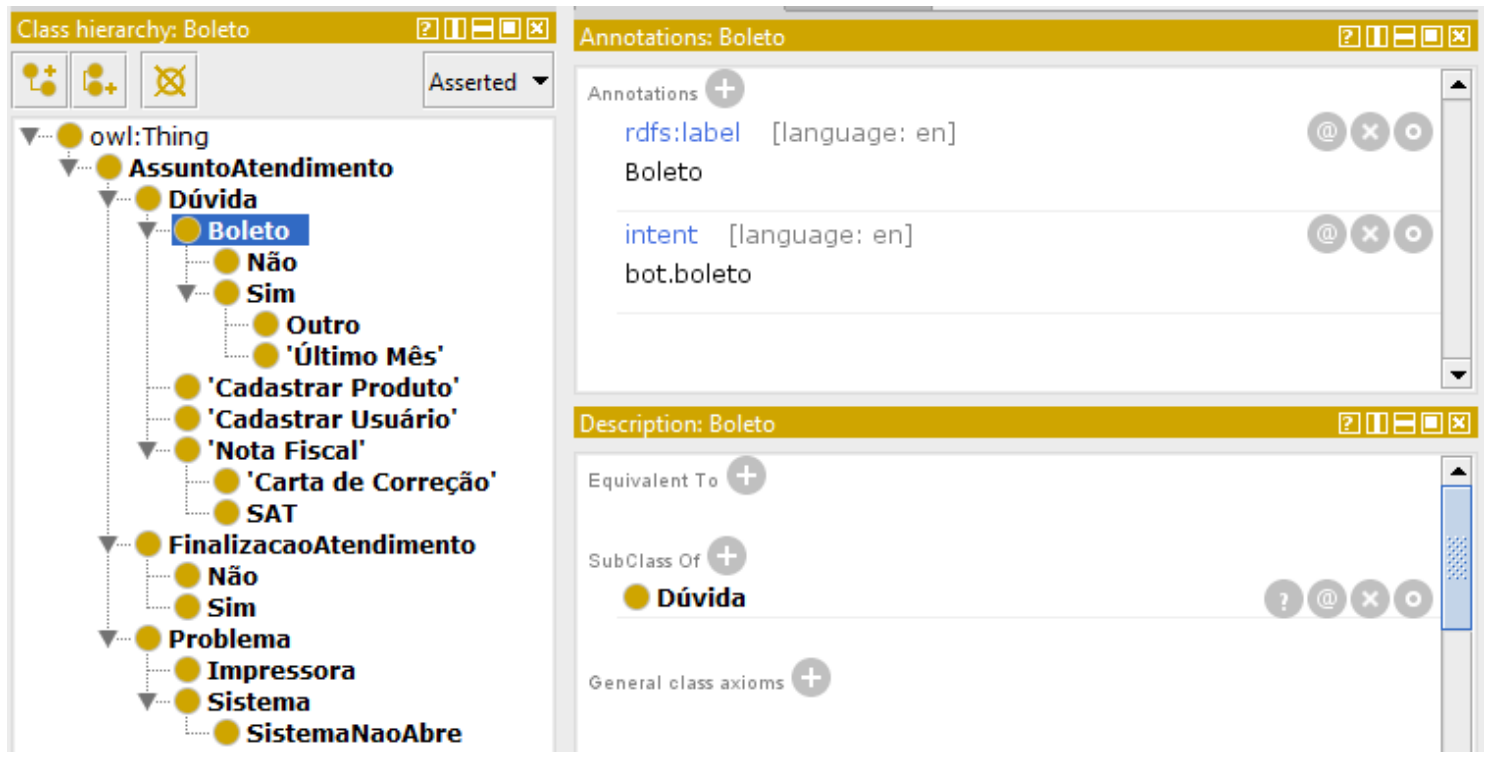

Figura 7. Modelo de ontologia utilizada no protótipo.

O servidor do chatbot envia uma requisição ao servidor de ontologia contendo o intent identificado pelo Dialogflow. O servidor de ontologia realiza uma consulta utilizando SPARQL [Pérez et al. 2009] para encontrar alguma classe com atributo intent igual ao que foi fornecido. Caso for encontrado, as subclasses imediatas são requisitadas. O re- 
sultado do webservice contém um vetor de string com o label das subclasses encontradas. As informações do Dialogflow e da ontologia são então combinadas e retornadas para a página web. Por fim a resposta e os botões de sugestão são exibidos para o usuário.

\section{Experimento}

Foi realizado um experimento com 10 usuários a fim de avaliar o chatbot integrado à ontologia utilizando os botões de sugestão. O roteiro do experimento consistiu em acessar um endereço web específico, realizar 3 sessões de interação com o chatbot e avaliar se o usuário utilizou os botões. Concluídos os experimentos, um questionário contendo duas perguntas objetivas e uma dissertativa foi aplicado aos usuários. Os dados foram obtidos por meio de log das conversas e comparando com os intents esperados em cada caso.

Cada uma das três sessões do experimento cobriu um caso específico de problema. O primeiro caso foi sobre senha de liberação, o segundo caso sobre segunda via do boleto da mensalidade do sistema e o terceiro caso sobre cadastro de um novo funcionário no sistema.

As perguntas objetivas abordam o tema de facilidade de uso do chatbot. A nota permitida varia de 1 a 5, sendo 1 "Discordo fortemente", 2 "Discordo", 3 "Neutro", 4 "Concordo" e 5 "Concordo fortemente". Dos 10 usuários avaliados as médias de notas para cada uma das duas perguntas objetivas foram 3.6 e 3.9. Os gráficos das respostas da questão 1 e da questão 2 podem ser vistos nas Fig. 8 e 9 respectivamente. Apenas um usuário respondeu à questão aberta sobre observações e críticas do projeto. Foi comentado que o chatbot identificou apenas um dos três casos.

O chatbot facilitou realizar as tarefas pedidas?

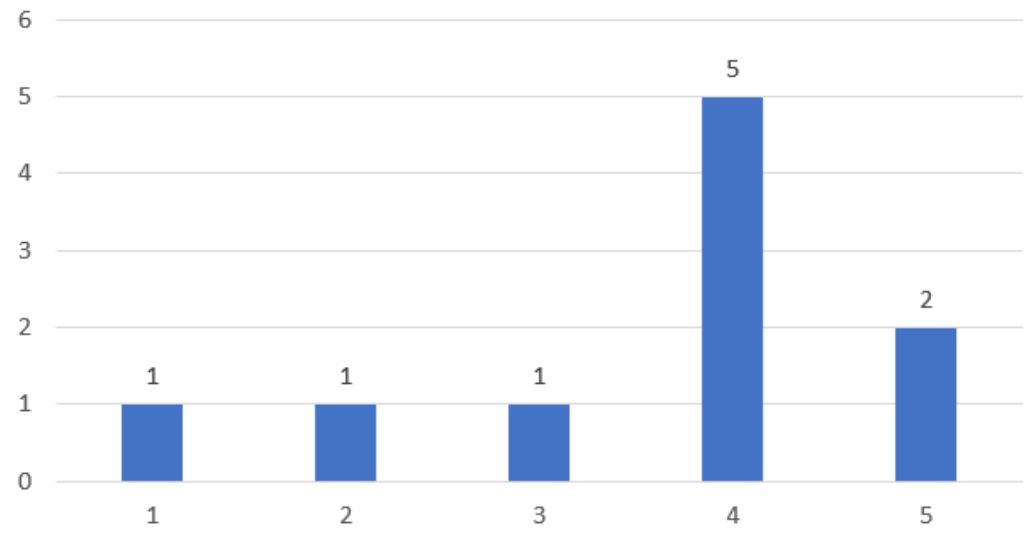

Figura 8. Gráfico da primeira questão objetiva.

Como pode ser observado na Tabela 1 , o caso 1 obteve um melhor desempenho pois houve um treinamento mais prolongado, em função do primeiro protótipo, com frases reais de usuários da empresa. Os casos 2 e 3 obtiveram um resultado menos satisfatório por conta de um número reduzido de frases de treinamento. Colunas marcadas com $\mathrm{S}$ significam que o chatbot interpretou corretamente a mensagem enviada pelo usuário e $\mathrm{N}$ significa que o usuário recebeu uma mensagem genérica e foi transferido para o suporte humano. 
As sugestões de resposta apresentadas pelo

chatbot facilitaram resolver as tarefas de forma

mais eficiente?

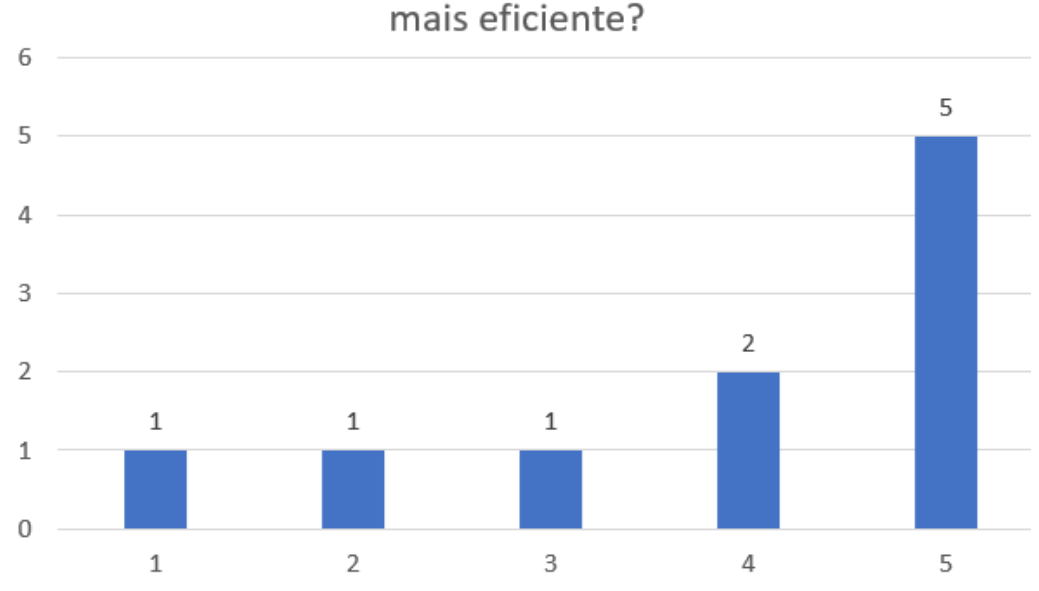

Figura 9. Gráfico da segunda questão objetiva.

Tabela 1. Resultado de experimento com usuários.

\begin{tabular}{|c|c|c|c|}
\hline & Caso 1 & Caso 2 & Caso 3 \\
\hline Usuário 1 & $\mathrm{S}$ & $\mathrm{N}$ & $\mathrm{N}$ \\
\hline Usuário 2 & $\mathrm{S}$ & $\mathrm{N}$ & $\mathrm{S}$ \\
\hline Usuário 3 & $\mathrm{S}$ & $\mathrm{N}$ & $\mathrm{N}$ \\
\hline Usuário 4 & $\mathrm{N}$ & $\mathrm{N}$ & $\mathrm{N}$ \\
\hline Usuário 5 & $\mathrm{S}$ & $\mathrm{N}$ & $\mathrm{S}$ \\
\hline Usuário 6 & $\mathrm{S}$ & $\mathrm{N}$ & $\mathrm{S}$ \\
\hline Usuário 7 & $\mathrm{S}$ & $\mathrm{S}$ & $\mathrm{N}$ \\
\hline Usuário 8 & $\mathrm{S}$ & $\mathrm{N}$ & $\mathrm{N}$ \\
\hline Usuário 9 & $\mathrm{S}$ & $\mathrm{S}$ & $\mathrm{S}$ \\
\hline Usuário 10 & $\mathrm{S}$ & $\mathrm{N}$ & $\mathrm{S}$ \\
\hline
\end{tabular}

Apenas duas do total de 30 sessões utilizaram o botão de sugestão de resposta, gerado a partir da interação com a ontologia. Uma hipótese para este resultado é de que a posição, forma ou cor dos botões não estão muito intuitivos, levando o usuário a desconsiderar o elemento da tela ou achar que não possui utilidade prática. Foi perguntado informalmente aos usuários após o experimento sobre os botões. A maioria disse não ter visto os botões na tela, o que corrobora a hipótese.

Devido ao pouco uso dos botões, não há dados suficientes para dizer se a integração com ontologia facilitou ou dificultou o uso do chat pelo usuário. Como trabalho futuro pode ser estudada a melhor forma de apresentar as sugestões ao usuário.

\section{Conclusões e Trabalhos Futuros}

Chatbots podem auxiliar a reduzir a quantidade de atendimentos em um ambiente de suporte online e consequentemente oferecer um melhor serviço ao cliente.

Foi criado um protótipo utilizando a plataforma [DIALOGFLOW 2018] com desempenho satisfatório para o caso de uso especificado. Uma versão modificada está sendo 
utilizada na empresa. Um segundo protótipo foi criado com base no primeiro utilizando ontologias para busca semântica e retorno de sugestão de respostas. O experimento com sugestão de resposta mostrou que não houve um uso significativo dos botões.

Como trabalho futuro pretende-se estudar formas de melhorar o engajamento do usuário com as sugestões de resposta.

\section{Agradecimentos}

Agradecimentos ao Programa Institucional de Bolsas de Iniciação Científica e Tecnológica do Instituto Federal de Educação Ciência e Tecnologia de São Paulo (PIBIFSP) - Edital 021/2017 - pelo auxílio financeiro a este trabalho de pesquisa. Agradecemos também à empresa JN Moura Informática pela parceria e disponibilização de dados e funcionários para a realização dos experimentos.

\section{Referências}

Al-Zubaide, H. and Issa, A. A. (2011). Ontbot: Ontology based chatbot. In International Symposium on Innovations in Information and Communications Technology, pages 7-12. Disponível em https://dx.doi.org/10.1109/ISIICT.2011. 6149594.

Carroll, J. J., Dickinson, I., Dollin, C., Reynolds, D., Seaborne, A., and Wilkinson, K. (2004). Jena: implementing the semantic web recommendations. In International World Wide Web Conference, pages 74-83. ACM. Disponível em https://doi . org/10.1145/1013367.1013381.

DIALOGFLOW (2018). Dialogflow. Disponível em https://dialogflow.com (Acesso em: 14 dez. 2018).

Knublauch, H., Fergerson, R. W., Noy, N. F., and Musen, M. A. (2004). The Protégé OWL Plugin: An open development environment for semantic web applications. In The Semantic Web - ISWC 2004, pages 229-243, Berlin, Heidelberg. Springer Berlin Heidelberg. Disponível em https://doi.org/10.1007/978-3-540-30475-3_ 17.

Pérez, J., Arenas, M., and Gutierrez, C. (2009). Semantics and complexity of SPARQL. ACM Trans. Database Syst., 34(3):16:1-16:45. Disponível em https : / / doi . acm . org/10.1145/1567274.1567278.

Shawar, B. A. and Atwell, E. (2007). Chatbots: are they really useful? Journal for Language Technology and Computational Linguistics, 22:29-49. Disponível em http://jlcl.org/content/5-allissues/ 19-Heft1-2007/Bayan_Abu-Shawar_and_Eric_Atwell.pdf.

Silva, M., Santos, F., Yaguinuma, C., and Boalim, T. (2018). Construção de ontologia de domínio combinada com expansão semântica de consultas para o desenvolvimento de um chatterbot. In III Encontro de Iniciação Científica e Tecnológica, Araraquara - SP.

WATSON (2018). IBM watson assistant. Disponível em https : / /www. i bm. com/ cloud/watson-assistant (Acesso em: 14 dez. 2018).

WITAI (2018). Wit.ai. Disponível em https : / /wit . ai / (Acesso em: 14 dez. 2018). 